\title{
Extreme events following bifurcation to spatiotemporal chaos in a spatially extended microcavity laser
}

\author{
S. Coulibaly, ${ }^{1, *}$ M. G. Clerc,${ }^{2, \dagger}$ F. Selmi, ${ }^{3}$ and S. Barbay ${ }^{3, \ddagger}$ \\ ${ }^{1}$ Laboratoire de Physique des Lasers, Atomes et Molécules, CNRS-UMR8523, Université de Lille 1, 59655 Villeneuve d'Ascq Cedex, France \\ ${ }^{2}$ Departamento de Física, Facultad de ciencias Físicas y Matemáticas, Universidad de Chile, Casilla 487-3, Santiago, Chile \\ ${ }^{3}$ Centre de Nanosciences et de Nanotechnologies, CNRS, Université Paris-Sud, Université Paris-Saclay, \\ C2N - Marcoussis, 91460 Marcoussis, France
}

(Received 18 August 2016; published 8 February 2017)

\begin{abstract}
The occurrence of extreme events in a spatially extended microcavity laser has been recently reported [Selmi et al., Phys. Rev. Lett. 116, 013901 (2016)] to be correlated to emergence of spatiotemporal chaos. In this dissipative system, the role of spatial coupling through diffraction is essential to observe the onset of spatiotemporal complexity. We investigate further the formation mechanism of extreme events by comparing the statistical and dynamical analyses. Experimental measurements together with numerical simulations allow us to assign the quasiperiodicity mechanism as the route to spatiotemporal chaos in this system. Moreover, by investigating the fine structure of the maximum Lyapunov exponent, of the Lyapunov spectrum, and of the Kaplan-Yorke dimension of the chaotic attractor, we are able to deduce that intermittency plays a key role in the proportion of extreme events measured. We assign the observed mechanism of generation of extreme events to quasiperiodic extended spatiotemporal intermittency.
\end{abstract}

DOI: 10.1103/PhysRevA.95.023816

\section{INTRODUCTION}

Macroscopic systems maintained out of equilibrium under the influence of injection and dissipation of energy, momenta, and particles are known to exhibit self-organization phenomena [1-4]. Usually, self-organization results from the interplay between linear gain and nonlinear saturation mechanisms. For small energy injection and large dissipation, one expects commonly to find a uniform steady state as global equilibrium. As one increases the injection of energy, this stable state suffers from instabilities which generate the emergence of oscillations or spatial structures [1-3]. Upon further increase of energy injection, one expects the system to display secondary instabilities, which can generate even more complex spatiotemporal behavior [5-7]. This is characterized by a complex spatiotemporal dynamics as result of the continuous coupling between modes in space and time [8]. Complex spatiotemporal dynamics has been observed in extended systems, for instance, in fluids [9-12], chemical reaction-diffusion systems [13], cardiac fibrillation [14], electroconvection [15], fluidized granular matter [16], and a liquid crystal light valve [17-19]. In these experimental observations, the scenarios or routes leading to spatiotemporal complexity is not fully understood in the context of macroscopic systems maintained out of equilibrium since there is not a well-established qualitative theory of spatiotemporal chaos for partial differential equations. In most of these studies, complex behaviors are characterized by the tools of dynamical system theory such as spatial and temporal Fourier analysis, wave-vector distribution, filtering of spatiotemporal diagrams, length scale distributions, Poincaré maps, number of defects as a function of parameters, largest Lyapunov exponent, and Lyapunov spectrum [9-19].

\footnotetext{
*saliya.coulibaly@phlam.univ-lille1.fr

${ }^{\dagger}$ marcel@dfi.uchile.cl

${ }^{\ddagger}$ sylvain.barbay@c2n.upsaclay.fr
}

In recent years, great effort has been devoted to the understanding of the observed large deviations in extended deterministic systems, the so-called extreme events. As defined in Ref. [20], a time recording of the dynamics of a macroscopic systems maintained out of equilibrium may consist of well-defined periods where a relevant variable undergoes small variations around a well-defined level defined by its long-time average. Occasionally, abrupt excursions to higher values that differ significantly from the average may appear, and these excursions are called extreme events. These events are ubiquitous in nature. In optics, an extreme event is characterized by a rare and intense optical intensity pulse. The study of extreme events and extreme waves [21] has been motivated by the analogy with rogue waves in hydrodynamics [22], giant waves recently observed in the ocean and whose formation mechanism is still not well understood. Physically, as a matter of fact, some conservative systems in optics and deep water waves in the ocean can share a common description by the nonlinear Schrödinger equation [23]. Most of the studies in this context have taken place in optical fibers where the interplay of nonlinearity, dispersion, and noise can generate extreme events [24-27]. Extreme events in conservative systems are often associated with the merging dynamics of coherent structures [28-30], and this mechanism has also been found in a dissipative, fiber-laser systems [25,31]. Other mechanisms observed in dissipative systems involve stochastically induced transitions in multistable systems [32] or the temporal chaotic dynamics in a nonspatially extended laser with optical injection [33]. Extreme events have been found in a variety of optical cavity systems, such as an injected nonlinear optical cavity [34], fiber lasers [31,35], solid-state lasers [36], optical liquid crystal light valve with optical feedback [37], and semiconductor lasers $[33,38]$. The role of spatial coupling has been studied in the context of a pattern-forming optical system subjected to optical feedback composed of a Kerr medium [39] or a photorefractive crystal [40] and in an extended microcavity 
laser with integrated saturable absorber [41]. In the latter observation, we have shown that extreme events emerge at the onset of spatiotemporal chaos, hence allowing us to connect the dynamical and statistical points of views. Our system is a planar vertical-cavity surface-emitting laser (VCSEL) cavity with integrated saturable absorber $[42,43]$ with a high aspect ratio, implementing a spatially extended nonlinear dissipative system. An interesting aspect of this system is that it does not display irregular dynamics and hence extreme events in the absence of spatial coupling. Spatial coupling comes from diffraction in the microcavity, which possesses a large Fresnel number and can henceforth accommodate a large number of transverse spatial modes. Experimental measurements in a quasi-one-dimensional configuration and numerical simulations allow us to identify the quasiperiodicity mechanism as the route to spatiotemporal chaos in this system. Moreover, by investigating the fine structure of the largest Lyapunov exponent, of the Lyapunov spectrum, and of the Kaplan-Yorke dimension of the chaotic attractor, we are able to infer that intermittency plays an important role in the variation of the number of extreme events found that correlates to the bifurcation of spatiotemporal chaos. As we will show, the mechanisms of spatiotemporal chaos appearance at stake here are extended quasiperiodicity route [44] and extended intermittence.

The characterization of chaos and spatiotemporal chaos can be achieved by means of Lyapunov exponents [45]. These exponents measure the exponential growth rate of generic small perturbations around of a given trajectory in finite-dimensional dynamical systems. There are as many exponents as the dimension of the system under study. When at least one exponent is strictly positive, an initial perturbation grows and the system diverges from its unperturbed trajectory: The system is chaotic. Additional information about the complexity of the system can be obtained from the exponents, for instance, the dimension of the strange attractor (spectral dimensionality), measures of the dynamic disorder (entropy) [46], or characterization of bifurcation diagrams [44]. Experimentally, one has access to the most dominant exponents only $[19,47]$. The analytical study of Lyapunov exponents is a thorny endeavor and in practice inaccessible. Hence, a reasonable strategy is to derive the exponents numerically by discretizing the mathematical model that accounts for the system under study. Let $N$ be the number of discretization points in an extended system described by $m$ real dynamical variables; then the system has $m N$ Lyapunov exponents $\lambda_{i}$. If the Lyapunov exponents are sorted in decreasing order and in the thermodynamic limit $(N \rightarrow \infty)$, these exponents converge to a continuous spectrum as conjectured by Ruelle [48]. Therefore, if the system displays spatiotemporal chaos in this limit, there exists an infinite number of positive Lyapunov exponents. The set of ordered Lyapunov exponents provides an upper limit for the strange attractor dimension through the Kaplan-Yorke dimension [46]

$$
D_{\mathrm{KY}}=p+\sum_{i-1}^{p} \frac{\lambda_{i}}{\lambda_{p+1}},
$$

where $p$ is the largest integer that satisfies $\sum_{i-1}^{p} \lambda_{i}>0$. In the thermodynamic limit, the Kaplan-Yorke dimension diverges with the size of the system as a consequence of the Lyapunov density [49].

The paper is organized as follows: In Sec. II, we describe the experimental setup and present the experimental results analyzed from a statistical and a dynamical point of views. In Sec. III we introduce a model for our one-dimensional spatially extended laser with a saturable absorber medium and analyze its phase diagram. In addition, we analyze the temporal evolution of the system from statistical and dynamical point of views and compare this with the experimental observations. The spatiotemporal chaotic behavior displayed by this model is characterized rigorously by obtaining the Lyapunov spectrum. The statistic of time differences between successive extreme events and spatiotemporal extended intermittency are also characterized. In Sec. IV we analyze the bifurcation of spatiotemporal chaos, looking at statistical and dynamical indicators. At last we conclude and give some perspectives.

\section{EXPERIMENTAL RESULTS}

To study how the number of extreme events following bifurcation of spatiotemporal chaos evolve in an extended system, we consider a planar vertical-cavity surface-emitting laser (VCSEL) with integrated saturable absorber and a high aspect ratio.

\section{A. Setup}

Figure 1(a) depicts the setup under consideration. The microcavity laser has a VCSEL-SA structure (vertical cavity surface emitting laser with intracavity saturable absorber) described in Refs. [42,43], already used to demonstrate bistable regimes, self-pulsing regimes (laser cavity solitons [43,50-52]), and excitable regimes [53,54]. It consists of two

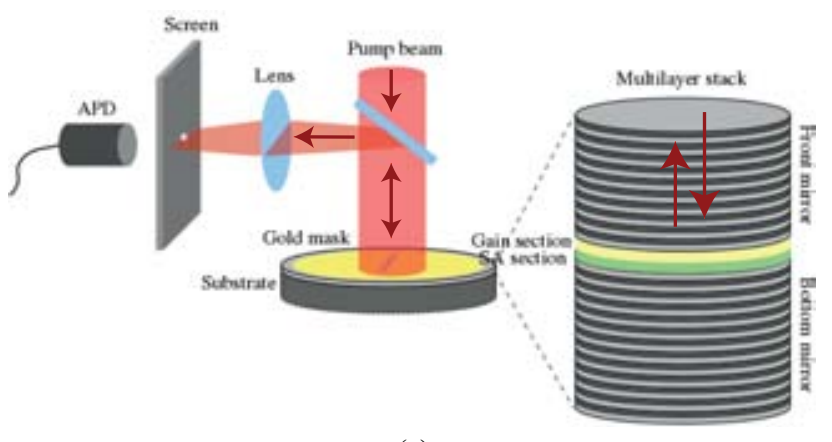

(a)

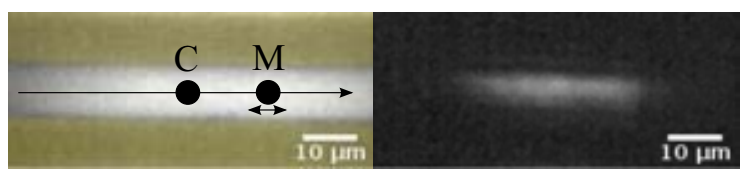

(b)

FIG. 1. (a) Schematic representation of the experimental setup (see text). (b) Top-view camera image of the one-dimensional (1D) line VCSEL-SA surface below (left) and above (lower image) laser threshold. The gold mask for the pump is colorized in yellow on the grayscale camera image to enhance visual contrast. 
(a)

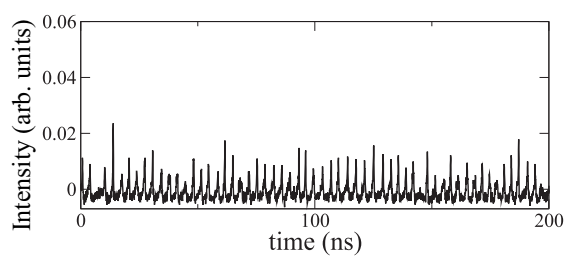

(b)

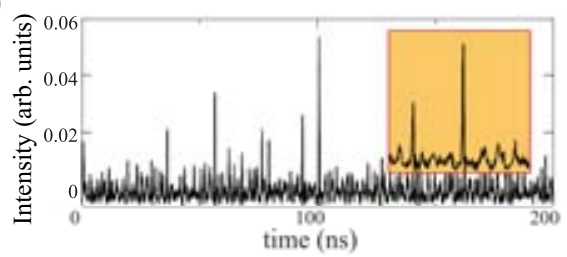

(c)

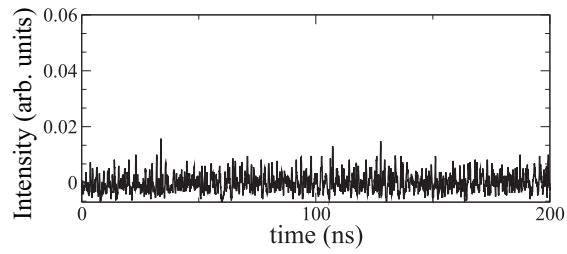

(d)

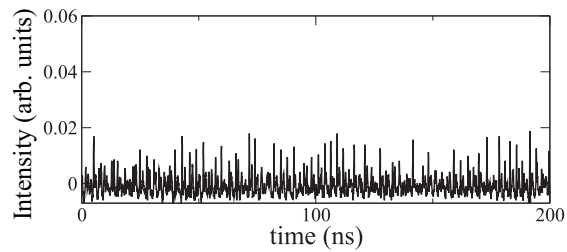

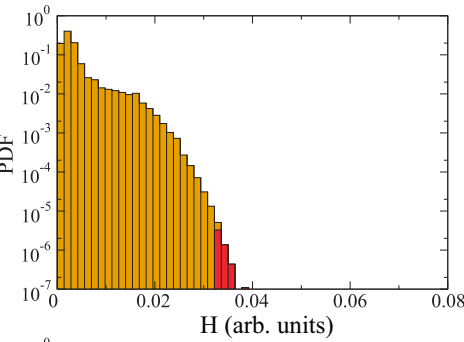
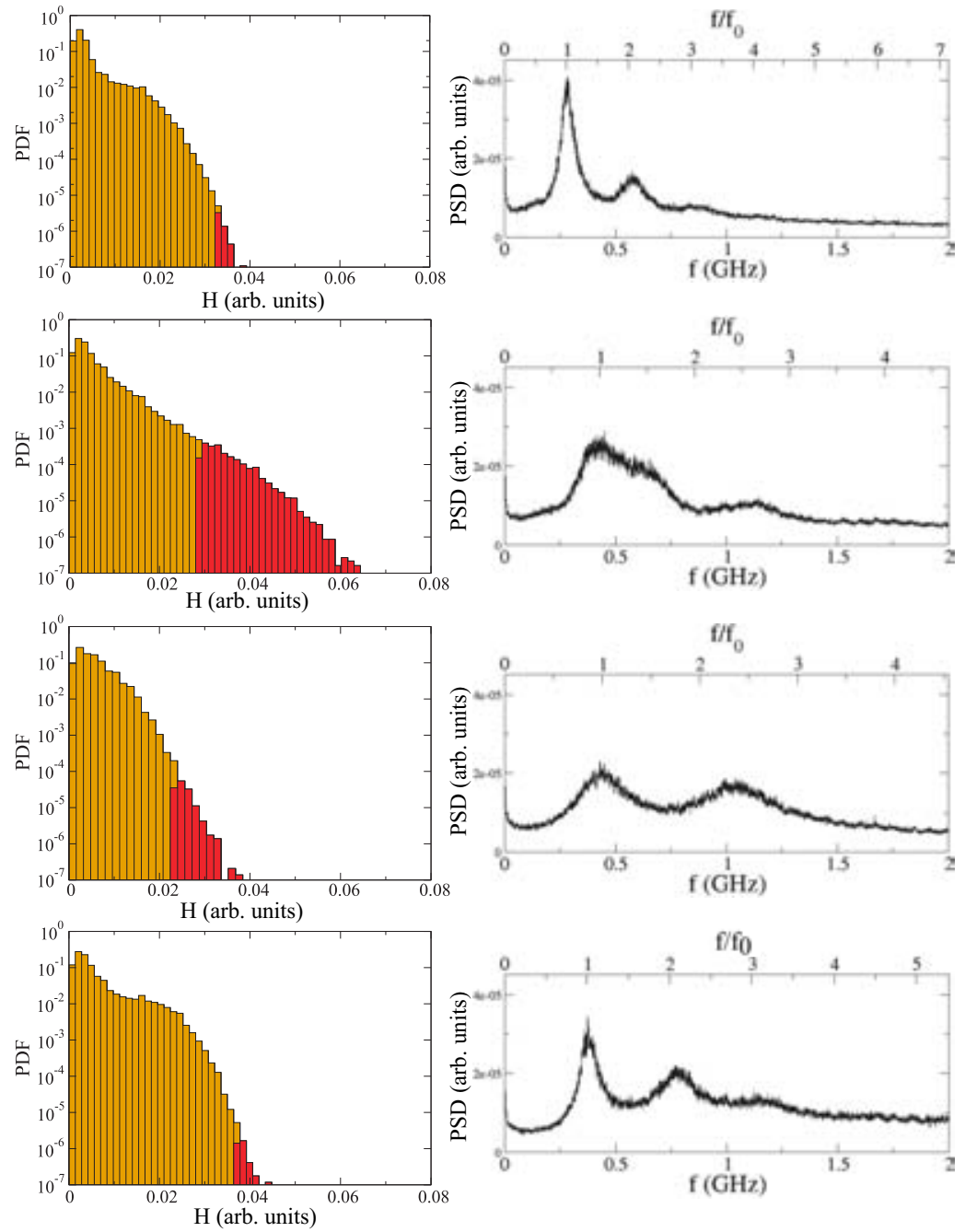

FIG. 2. Experimental observation of extreme events in a spatially extended microcavity laser. Excerpt of the temporal evolution of the intensity at position $\mathrm{C}$, corresponding logarithm of the PDF of the intensity height $H$ and power spectral density (PSD) for different normalized pump values $P / P_{\mathrm{th}}$ : (a) 1.02 , (b) 1.17 , (c) 1.20 , and (d) 1.25 . Extreme events $(A I>2)$ are emphasized in red.

specially designed multilayer mirrors for optimized optical pumping and an active zone with two InGaAs quantum wells for the gain section and one InGaAs quantum well for the saturable absorber (SA) section. The laser cavity resonance is targeted at $980 \mathrm{~nm}$ and the pump is optimized in a pumping window around $800 \mathrm{~nm}$. A high-power, fiber-coupled diode laser array provides the optical pumping at $974 \mathrm{~nm}$. The output of the multimode, $800-\mu \mathrm{m}$ diameter fiber is imaged with a microscope objective $(\times 20)$ onto the VCSEL-SA surface to form a top-hat-shaped pumping profile. A gold mask is deposited onto the sample surface to allow for different pump geometries. We concentrate on an elongated-shaped pump profile having a rectangular opening $80 \mu \mathrm{m}$ long and $10 \mu \mathrm{m}$ wide [Fig. 1(b)], thus forming a line laser and a quasi-onedimensional geometry. A dichroic mirror allows us to separate the excitation wavelength from the laser emission. Laser emission is imaged with a telescope arrangement (represented by one lens in Fig. 1 for simplicity) on a screen and on a camera sensor plane. The screen is provided with one or two holes for spatial selection of the detection area. It corresponds roughly to selecting a 5- $\mu \mathrm{m}$-diameter disk on the sample surface. The same detection arrangement is duplicated if two different areas are to be simultaneously recorded. The line VCSEL-SA emission intensity is recorded with a fast avalanche photodiode (APD, $5 \mathrm{GHz}$ bandwith). The temporal signal is amplified in a low-noise, high-bandwith amplifier (Miteq model JSMF302K180-30-10P, $3 \mathrm{kHz}-18 \mathrm{GHz}$ bandwidth) and acquired with a $6-\mathrm{GHz}$ oscilloscope at $20 \mathrm{GS} / \mathrm{s}$. Up to $50 \times 10^{6}$ points can be acquired in a single trace. The near field of the laser below and above threshold is shown in Fig. 1. Time traces, once acquired, are treated to display the histogram of the peak intensities.

\section{B. Temporal, statistical, and spectral analyses}

To characterize the complex spatiotemporal dynamics exhibited by the microcavity laser, we have monitored the temporal evolution of the intensity at the center of the laser (position $\mathrm{C}$ ) and computed the logarithm of the probability 
distribution function (PDF) of the intensity height $H$ and the Fourier spectrum. Figure 2 shows representative temporal evolution of the total intensity, corresponding logarithm of the intensity height histograms, and their Fourier spectrum for different values of the pump. Regular dynamical behaviors such as oscillations or quasiperiodicity show a quite simple temporal evolution and Fourier spectrum [55]. Then, the Fourier spectrum is characterized by some modes and their harmonics. This scenario changes drastically when one has complex behaviors like chaos or spatiotemporal chaos. The Fourier spectrum exhibits the coupling of many modes. For normalized pump power parameter close to laser threshold, $P / P_{\text {th }}=1.02$, where $P_{\text {th }}$ is the pump at laser threshold, the system exhibits a quasiperiodic behavior for the wave envelope; i.e., the dynamics of the pulse train amplitude is harmonic, as shown in the top panel of Fig. 2. The power spectrum exhibits a well-defined frequency and its secondary harmonics [cf. right panel of Fig. 2(a)]. The peak broadening is due to fluctuations inherent to the system. In this parameter regime the PDF of the intensity height is characterized by a quadratic decay in the tail in a semilog plot; i.e., this distributions presents a Gaussian tail and looks like a Rayleigh distribution for a positive-valued Gaussian process [see middle panel of Fig. 2(a)]. We have computed the threshold for extreme events as follows. We take the traditional criterion considering extreme events as those having a height $H$ twice the significant height $H_{s}$ (mean of the highest tercile of the PDF), i.e., with an abnormality index $A I=H / H_{s}>2$. The height $H$ is extracted as the maximum of the left $H_{l}$ and right $H_{r}$ heights $H=\max \left(H_{l}, H_{r}\right)$. Note that taking either $H, H_{l}$, or $H_{r}$ as the height does not change the results significantly. To get rid of the large number of small peaks of detection noise at the left of the PDF, we compute the significant height $H_{s}$ only by considering events whose height is larger than the observed maximum peak-to-peak noise amplitude, which is about $5 \mathrm{mV}$ (note that the rms noise is only $0.9 \mathrm{mV}$ ). This threshold introduces a more stringent criterion for extreme events detection. Extreme events are depicted in red under the histograms presented in Fig. 2. We observe, as expected, that the maximum number of extreme events is obtained in the PDF with a non-Gaussian tail, i.e., with a normalized pump of 1.17. As the pump is increased, the system exhibits complex behavior characterized by intermittent pulsation of total intensity [see left panel of Fig. 2(b)]. This dynamic is characterized by irregular oscillation of intensity, exhibiting sharp beats that appear in an aperiodic manner. The statistics of the intensity heights develops long tails with an initial exponential decay and the PDF shows signs of fat tails with positive deviations from the exponential decay $\left(P / P_{\text {th }}=\right.$ 1.17). The middle panels of Fig. 2 depict the regular and extreme events with different colors. Likewise, its respective power spectrum shows that frequency peaks spread and mix, which is a typical signature of chaotic behavior [55]. These observations allow us to infer that the dynamics exhibited by the system is a natural spatial extension of supercritical intermittency route to chaos [56]. As we will see later, this dynamic is described mathematically by coupling of large number of spatial modes, which exhibit a continuous Lyapunov spectrum [41]. Under further increase of the pump power $\left(P / P_{\text {th }}=1.20\right)$, intermittent dynamic behavior in the temporal

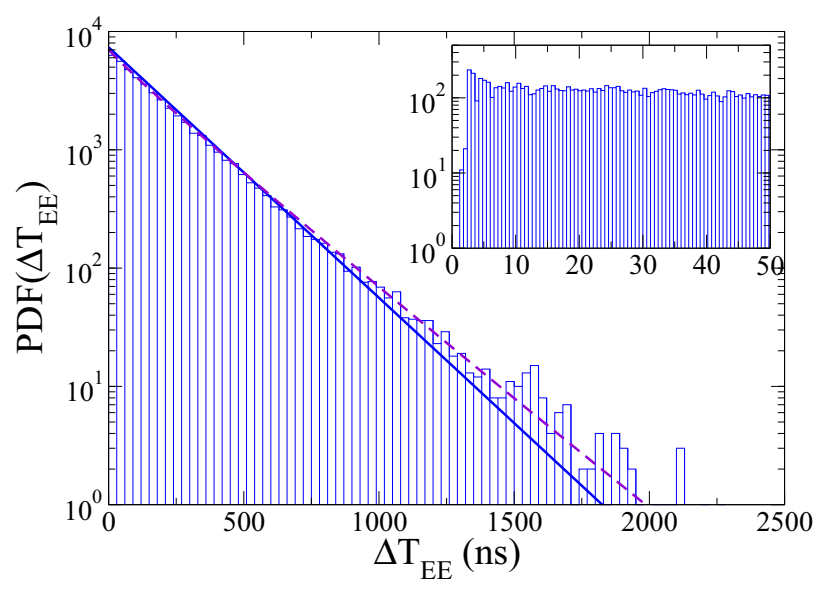

FIG. 3. Histogram of time differences between successive extreme events $\Delta T_{\mathrm{EE}}$ for $P / P_{\mathrm{th}}=1.17$, which corresponds approximately to a Poisson statistics with exponential behavior (full line) characterized by a mean $\sigma=205 \mathrm{~ns}$. A stretched exponential distribution function of the form $e^{-(t / \sigma)^{b}}$ with $b=0.95$ is also shown for comparison (dashed line). In the inset is shown a closeup of the histogram for small time difference (with higher binning).

evolution of the total intensity is replaced by irregular oscillations of the total intensity, which does not exhibit extreme peak intensities [cf. Fig. 2(c)]. The statistics of the intensity heights continues to present an exponential tail with rare events; however, the tail size has been reduced substantially. Notwithstanding, the power spectrum analysis reveals that the observed dynamic behavior is of quasiperiodic type with the presence of noiselike fluctuations. It is important to note that the dominant observed frequencies seem incommensurable, in the limits of the experimental accuracy. Hence, this evidences that quasiperiodic behavior with fluctuations may display rare events. For still higher pump values $\left(P / P_{\text {th }}=1.25\right)$, the temporal evolution of the intensity shows aperiodic behavior. However, the observation of large pulses is very rare. Figure 2(d) shows the typical temporal dynamics. The statistics for high amplitude is Gaussian-like. Notice that the mean pulse period decreases with pump as expected in a zero-dimensional laser with saturable absorber, and because of the spatial coupling, the intensity is very irregular and displays a complex dynamics (cf. Fig. 2). Close to the Hopf bifurcation, where the oscillatory instability disappears, the oscillation amplitude decreases and the oscillation becomes more harmonic since the effect of the nonlinearities decreases.

To figure out the statistics of extreme events appearance in time, we show in Fig. 3 the histogram of times between two successive extreme spikes with $A I>2$, corresponding to Fig. 2(b). It displays Poisson-like statistics characterized by an exponential decrease with mean interspike time $\sigma=205 \mathrm{~ns}$. Hence we can conclude that in the first approximation extreme spike appearance obeys a Poisson, memoryless process; that is, each rare event is temporally independent from the others. At very small times (inset of Fig. 3) there is a deviation from the Poisson statistics with lower probability to observe an extreme event immediately after or before one has already been observed. This can be understood by looking at the average 

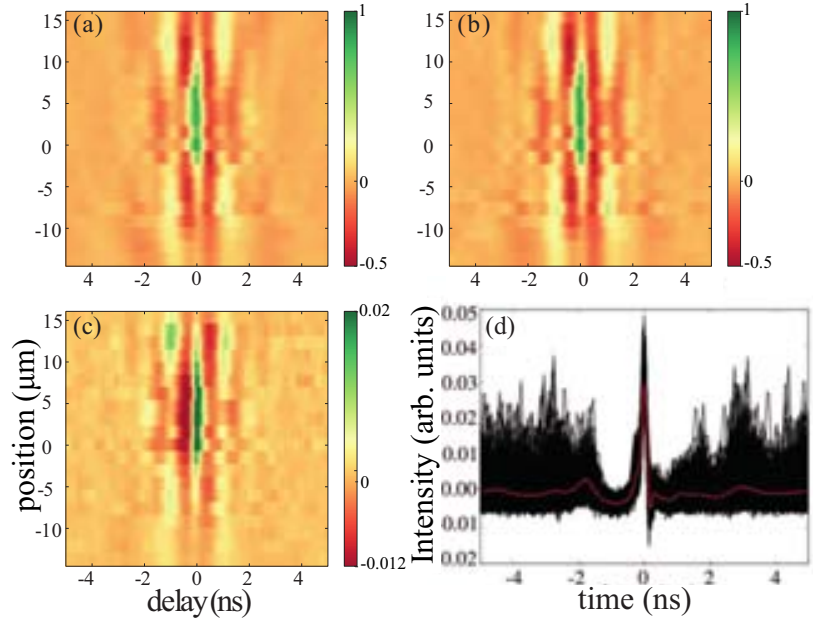

FIG. 4. (a) Temporal cross correlation $X_{c, m}\left(t_{k}, x_{m}\right)$ (see text) between the detector responses in points $\mathrm{C}$ (position $x_{m}=0$ ) and $\mathrm{M}$ at delays $t_{k}=k \Delta t$. (b) Same as in panel (a), restricted to extreme events at point $C$. (c) Average of the responses at point $M$ and at times where an abnormal event has occurred in the center of the laser in $\mathrm{C}$. The pump in panels (a)-(c) is $P / P_{\text {th }}=1.20$. (d) Average over 1000 extreme events in $\mathrm{C}$ for $P / P_{\mathrm{th}}=1.17$. The extreme events considered are superimposed and plotted in black, and the mean is in red.

shape of an extreme event in Fig. 4(d). Correspondingly, there is a higher probability than the Poisson statistics to observe an extreme event about 2 ns before or after a given extreme event. This phenomenon could be related to the three sisters effect [57]. For very large time differences, there is also a small deviation from the Poisson statistics that is well fitted by a stretched exponential distribution function of the form $e^{-(t / \sigma)^{b}}$ with stretching coefficient $b=0.95$. The PDF of the time difference between extremes has been studied in the context of the classical extreme value theory in dynamical systems and the first return time statistics has been shown to be distributed according to a Poisson law [58]. However, most of these classical theories neglect possible correlation among extremes and some authors showed that in that case, a stretched exponential distribution in expected $[59,60]$. Our result is thus in accordance with the expected statistics of the first return times in the case where there are very long-term correlations in the occurrence of extreme events, as is often observed. Interestingly, our result is in contrast to what has been reported in other optical systems in, e.g., Refs. [21,38], where a log-Poissonian distribution is found. This kind of distribution has been found also in dissipative systems containing many attractors in the presence of noise [61]. We therefore attribute the differences in the PDF of the return times statistics to the specific process that generates extreme events and to the possible correlations that result. This latter point would deserve further investigation to clarify the role of long-range correlations and is postponed to future work.

\section{Statistical spatiotemporal analysis}

We now study the spatiotemporal structure of the statistics of emitted pulses. We record the dynamics in two points of the experimental setup, one at a fixed position at the center of the laser and the other moving along the long line laser. In Fig. 4(a), we plot the normalized cross correlation $X_{c, m}(k)$ of the $N=100000$ first recorded points $(5 \mu \mathrm{s})$ between the signal recorded at the central detector $y_{c}$ at point $C$ and the one at the moving detector $y_{m}$ at location $\mathrm{M}, 1 \leqslant m \leqslant 20$ such that

$$
X_{c, m}(k)=\frac{1}{N \sigma_{y_{c}} \sigma_{y_{m}}} \sum_{i}\left[y_{c}(i)-\bar{y}_{c}\right]\left[y_{m}(i+k)-\bar{y}_{m}\right],
$$

where the bar symbol indicates the mean value and $\sigma$ is the standard deviation. In the central part appears a zone with high positive (green) cross correlation followed and preceded by two bands of negative cross correlation. The temporal band in which the cross correlation is nonzero extends about 2 ns from around zero delay. This demonstrates that there is indeed a finite spatial correlation length in the system and that this correlation length is smaller than the system size. However, since the correlation bands are vertical at these time scales, there is no clear evidence of propagation effects (at least with the temporal resolution of our setup) though there is a slight bending of the correlated band (in green). In Fig. 4(b) we restrict the cross correlation around the points where $A I>2$; i.e., we consider only extreme events. We notice that there are no major differences between the two cross correlations; hence there seems not to be any statistical marker of the appearance of an extreme event in this regime, and in particular no clear sign of propagation of a coherent structure either. Indeed, these results indicate that extreme height intensity peaks appear in a spatial correlation zone and disappear almost immediately everywhere in this zone. Correlation is therefore maximum at zero delay for almost all positions detected. Figure 4(c) shows the average of the responses at position $\mathrm{M}$ and at times where an abnormal event has occurred in the center of the laser in $\mathrm{C}$. The average shows a clear time asymmetry around the correlated structure: Every selected event begins with a large amplitude dip followed by a large positive peak. This can also be seen in Fig. 4(d). On the wings of the correlated zone we can see another dip.

\section{NUMERICAL SIMULATIONS OF A ONE-DIMENSIONAL SPATIALLY EXTENDED LASER WITH A SATURABLE ABSORBER MEDIUM}

We can compare our results with numerical simulations of an existing model of a one-dimensional spatially extended laser with a saturable absorber (LSA) medium [62]. The model consists in three coupled nonlinear partial differential equations

$$
\begin{aligned}
\frac{\partial E}{\partial t} & =[(1-i \alpha) G+(1-i \beta) Q-1] E+i \frac{\partial^{2} E}{\partial x^{2}}, \\
\frac{\partial G}{\partial t} & =\gamma_{g}\left[\mu-G\left(1+|E|^{2}\right)\right], \\
\frac{\partial Q}{\partial t} & =\gamma_{q}\left[-\gamma-Q\left(1+s|E|^{2}\right)\right],
\end{aligned}
$$

for the intracavity electric-field amplitude $E(x, t)$ and the carrier density in the gain (saturable absorber) medium $G(x, t)$ $[Q(x, t)]$. The nonradiative carrier recombination rates are $\gamma_{g}$ 


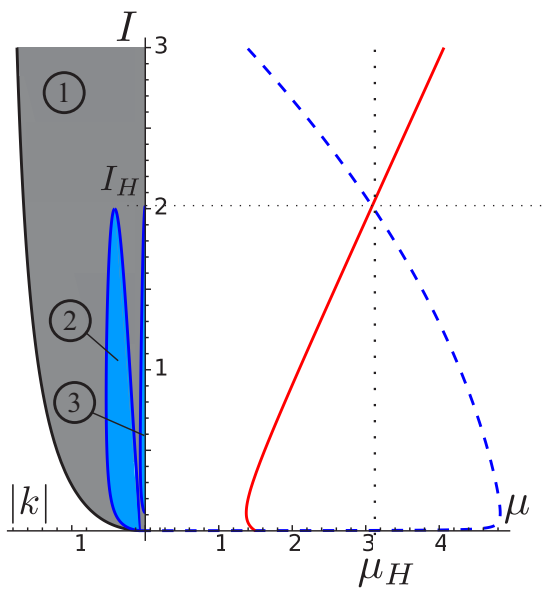

FIG. 5. Bifurcation diagram of the one-dimensional spatially extended laser with a saturable absorber media described by Eqs. (3). Characteristic curve $\mu(I)$ (full line, red) along with the unstable wave-vector regions of the the linear stability analysis [Turing instability, gray (1); Andronov-Hopf instability, blue (2-3)]. Right axis is $\mu$ and left axis is the modulus $|k|$ of the unstable wave vectors. The plane-wave Hopf curve is shown by the dashed blue line.

and $\gamma_{q}$ with pumping $\mu$ and linear absorption $\gamma$. The Henry enhancement factors in both sections are $\alpha$ and $\beta$ respectively. Diffraction is included through the complex Laplacian term. Note that the processes of diffusion of carriers is neglected in this model. Time has been rescaled to the field lifetime in the cavity, which is calculated to be here 8.0 ps given the cavity design parameters. Space is rescaled to the diffraction length $w_{d}$, which is $7.4 \mu \mathrm{m}$. We take parameters compatible with our semiconductor system: $\alpha=2, \beta=0, s=10, \gamma_{g}=$ $\gamma_{q}=0.005$, and $\gamma=0.5$. The $\alpha$ and $\beta$ parameter are chosen with standard values [63]. Their values obviously influence the instability range [62] but do not change the main conclusions of this work. The other parameters are deduced from typical physical values for this semiconductor structure, giving recombination times of carriers of the order of $800 \mathrm{ps}$. In order to take into account the stiffness of the problem for these parameters, the implicit Runge-Kutta method RADAU5 [64] is used for numerical integration. The width of the integration region $w$ is $w / w_{d}=24$ with a top-hat pumping of width $w_{p} / w_{d}=12$. Based on the results developed in Ref. [62], we can describe the main properties of the stationary solutions and the linear stability analysis. The system has a first threshold for $\mu_{\mathrm{th}}=1+\gamma$. The plane-wave characteristics curve of the laser is given by $\mu /(1+I)-\gamma /(1+s I)=1$, which gives a $\mathrm{C}$-shaped curve with a subcritical bifurcation at threshold provided $s>1+1 / \gamma$. For a certain range of parameters, the system also exhibits an Andronov-Hopf bifurcation for plane waves in a given intensity range $I$ such that $\mu(I)<\mu_{H}(I) \equiv r\left[2 r s I \gamma-\gamma_{g}(1+I)(1+s I)(1+\right.$ $I+r+r s I)] / 2 I$ with $r=\gamma_{q} / \gamma_{g}$. When including the spatial degree of freedom, a linear stability analysis can be performed by checking the stability of Eqs. (3) with respect to small harmonic perturbations of the form $\exp (\lambda t+i k x)$. The results are summarized in Fig. 5 for the latter set of parameters. It reveals that the upper branch is usually Turing unstable everywhere, hence with a band of unstable wave vectors $k$ with a real and positive growth rate $\lambda$ (region 1 in Fig. 5), giving rise to a complex spatiotemporal dynamics. An Andronov-Hopf instability can also occur either for the plane-wave case or triggered by the spatial coupling. They are associated respectively to the regions 1 and 2 in Fig. 5.

\section{A. Temporal, statistical, and spectral analysis of numerical simulations of a one-dimensional spatially extended LSA model}

One can see that region 1 in Fig. 5 extends to infinite intensities, meaning that the whole branch is modulationally unstable. The plane-wave Andronov-Hopf unstable curve intersects the characteristic curve at $\mu=\mu_{H} \sim 3.08$ resulting in a self-pulsing behavior below $\mu_{H}$ for the nonspatially extended system in the well-known scenario already described in, e.g., Ref. [65]. There is a range of wave vectors for which harmonic spatial perturbations are linearly unstable for intensities below $I_{H}$. For the set of parameters we choose we have no Andronov-Hopf instabilities excited by spatial coupling alone.

To characterize the dynamics exhibited by the onedimensional spatially extended laser with saturable absorber medium from the statistical and dynamical point of views, we have numerically simulated the set of Eqs. (3) and considered different values of power pump parameter $\mu$. For small pumping values, $\mu<\mu_{\text {th }}$, the nonlaser state is stable. When increasing the pump power parameter above threshold $\left(\mu>\mu_{\mathrm{th}}\right)$, the laser turns on through a transcritical bifurcation [66] and the system exhibits plane waves with oscillations in its envelope. The left panel of Fig. 6(a) shows the temporal evolution of the total intensity. To grasp this oscillation, we have calculated its power spectrum. In middle right panel of Fig. 6(a) we present this power spectrum, where the dominant frequency and their harmonics are observed. In addition, we have reconstructed the oscillation in its phase portrait, using the embedding (or attractor reconstruction) method [47]. The left panel of Fig. 6(a) displays the reconstructed limit cycles obtained from the temporal evolution of the total intensity. We can see that the dynamics is composed of a small amplitude oscillation around the origin subsequently accompanied by a large amplitude oscillation (the intensity pulse). The limit cycle shape is typical of an attractive periodic solution that emerged from a homoclinic Shilnikov bifurcation [67]. It is well known that model Eq. (3) has a homoclinic bifurcation, which is consistent with the observed limit cycle type [65]. The statistics of the local intensity height $I_{\mathrm{loc}}$ is obtained from the space time evolution (as shown in Fig. 9), in a similar fashion as reported in Ref. [68] in the 1D propation in an optical fiber, a method recently extended to $2 \mathrm{D}+1$ space-time evolution in a laser with saturable absorber [69]. A peak is found for a simultaneous maximum of the intensity in space and time. The PDF of $I_{\text {loc }}$ has a bimodal structure that accounts for both oscillations [cf. left middle panel of Fig. 6(a)].

By increasing the pumping value $\left(\mu / \mu_{\mathrm{th}} \sim 1.047\right)$, the total intensity exhibits a quasiperiodic behavior; i.e., the dynamics is aperiodic and shows fluctuations around a constant value of the total intensity. The left panel of Fig. 6(b) depicts the temporal evolution of total intensity. To infer the dynamics observed, in the middle right panel of Fig. 6(b) we show the 

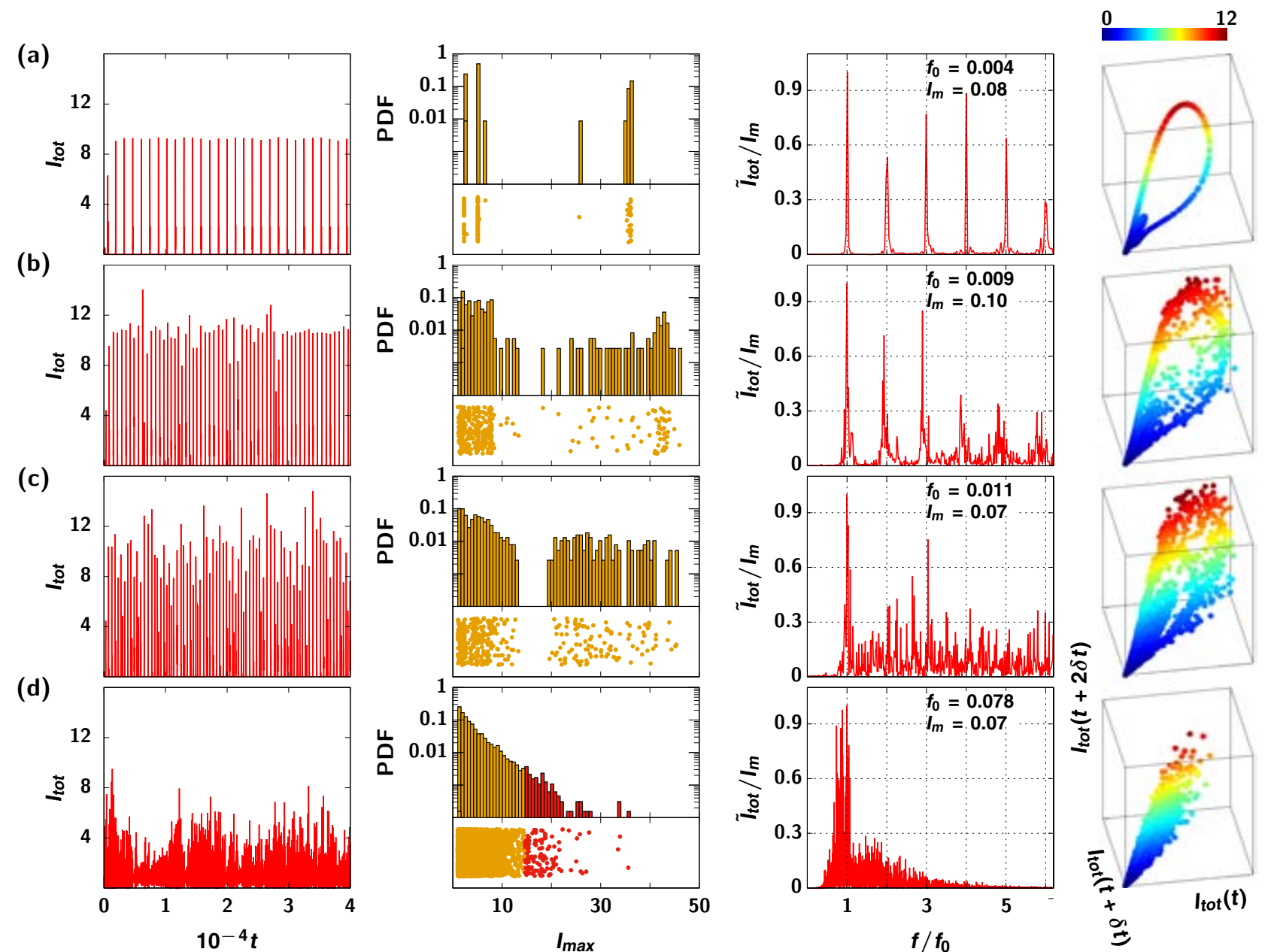

(d)
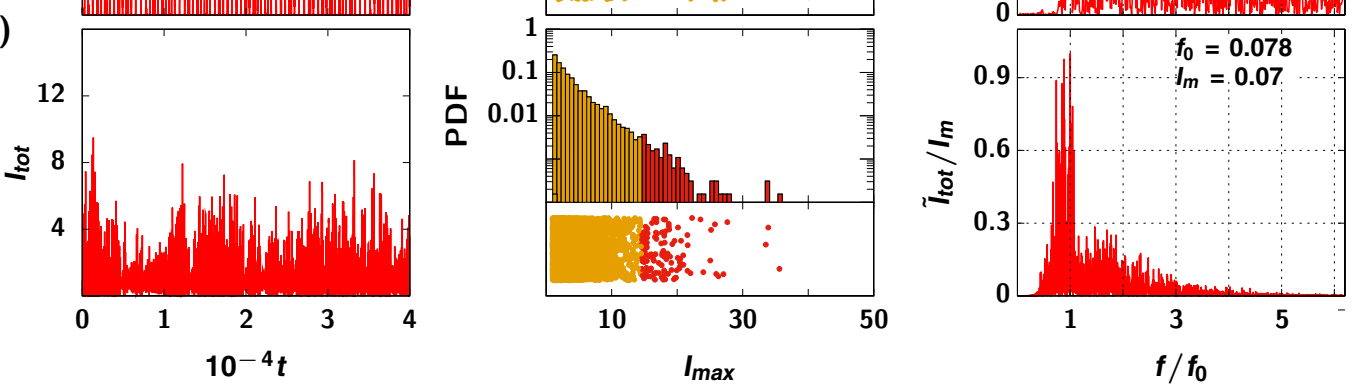

FIG. 6. Numerical observation of extreme events in a one-dimensional spatially extended laser with a saturable absorber medium, Eq. (3), with $\alpha=2, \beta=0, \gamma_{g}=0.005, \gamma_{q}=0.005, \gamma=0.5$, and $s=10$. Columns account for excerpts of the temporal evolution of total intensity, corresponding logarithm of the PDF of the theoretical local intensity height $I_{\text {loc }}$ (local maxima of the spatiotemporal intensity map), the Fourier spectrum, and phase space embedding reconstruction for different normalized pump values $\mu / \mu_{\mathrm{th}}$ : (a) 1.017, (b) 1.047, (c) 1.053, and (d) 1.33. Extreme events $(A I>2)$ are emphasize in red (light gray) in the histogram. At the bottom of each histogram is a scatter plot where the points account for the observations of their respective amplitude (vertical axis is arbitrary). The color code in the phase space diagram keeps track of the respective intensity values.

associated power spectrum. From this spectrum, one deduces that the observed dynamics is of quasiperiodic type [45]. This is because one clearly observes frequencies that are not commensurable. The reconstruction of the phase portrait shows that the limit cycle is replaced by a torus. The logarithm of the PDF of $I_{\text {loc }}$ has an $L$ shape [cf. left middle panel of Fig. 6(b)]. This structure is a result of the deformation of the bimodal distribution. However, extreme events are not observed in this region of parameters with our criterium, and this is in good agreement with what one could expect from the temporal evolution. By further increasing the pumping parameter $\left(\mu / \mu_{\mathrm{th}} \sim 1.053\right)$, the temporal evolution of the total intensity is qualitatively similar. The power spectrum is increasingly enriched by the presence of more modes; however, the behavior remains of quasiperiodic type. The torus obtained in the reconstructed phase portrait increases in size.
The logarithm of the PDF of $I_{\text {loc }}$ remains qualitatively similar too. Figure 6(c) illustrates all these results.

Continuing to increase the value of the pumping parameter $\left(\mu / \mu_{\mathrm{th}} \sim 1.333\right)$, the system changes qualitatively its dynamical behavior; i.e., it presents a bifurcation. The temporal evolution of the total intensity exhibits intermittent pulsation [see the left panel of Fig. 6(d)]. This dynamics is characterized by irregular fluctuations of intensity exhibiting sharp beats that appear in an aperiodic manner, which is consistent with the experimental observations in Fig. 2(b). The power spectrum is characterized with broad peaks, which are typical of complex behaviors such as chaos [45]. To figure out the nature of the complex dynamics observed, we have calculated the Lyapunov spectrum. Figure 7 shows the Lyapunov spectrum for several values of the pumping parameter $\mu$. This figure clearly shows that when the system exhibits extreme events it 


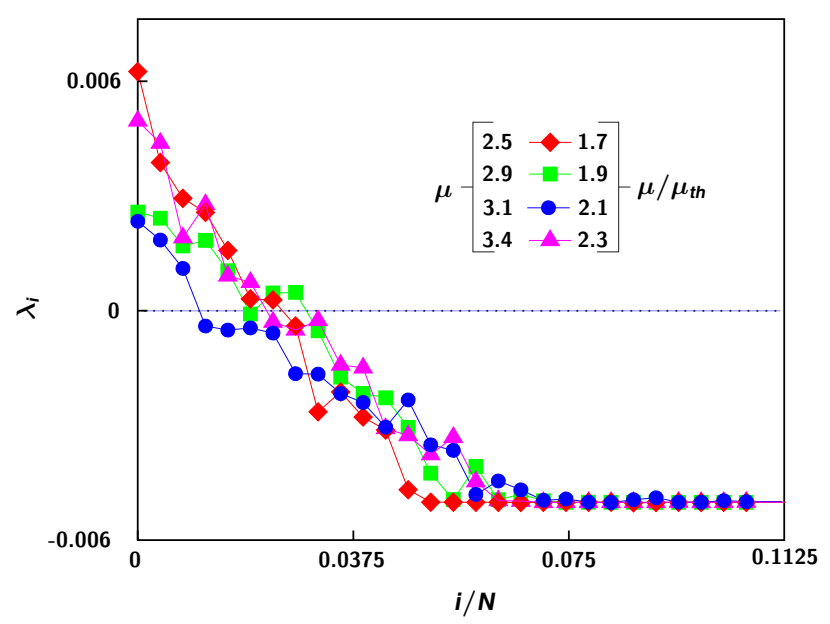

FIG. 7. Numerical Lyapunov spectrum of a one-dimensional spatially extended laser with a saturable absorber medium, Eq. (3), for different pump parameters $\mu$. The other parameters are the same as in Fig. 6 and $N=512$ is the total number of Lyapunov exponents computed.

is in a regime of spatiotemporal chaos with several nonzero Lyapunov exponents in the Lyapunov spectrum. The Lyapunov exponents were computed using the strategy proposed in Refs. [70,71]. We have reconstructed the respective attractor in phase space and a complex object is observed [see the right panel of Fig. 6(d)], which clearly cannot be embedded in three dimensions. That is, its geometrical characterization requires a higher embedding dimension. The logarithm of the PDF of $I_{\text {loc }}$ for the $1 \mathrm{D}$ laser with saturable absorber is shown in the left middle panels of Fig. 6(d). In this regime, the system displays a distribution with long and exponential decay, which is similar to the one found in Ref. [41] using the total intensity as an observable. Extreme events are depicted in dark color (red) under the histograms presented in the left middle panel of Fig. 6(d), and a lot of extreme events are observed in this regime. We note that the observed dynamic is in excellent agreement with experimental observations (cf. Figs. 2 and 6). The shape of the PDF of $H$ seems to be correlated to the presence or absence of a Andronov-Hopf bifurcation: Only when it is present can we observe a heavy-tailed distribution. At the transition between the Hopf-Turing and Turing-only regions we observe that a maximum number of extreme events are generated (for $\mu=2.9$ ).

We have conducted an analysis of the return time statistics between extreme events. Figure 8 summarizes the main features of the observed dynamics. The histogram of time differences between successive extreme events (or waiting time) is represented in Fig. 8(b). As noted earlier, the observed temporal dynamics is not completely described by a Poisson process, but is rather well described by a stretched exponential distribution function with stretching parameter equal to 0.5 . To highlight the differences between these processes we have calculated the cumulative distribution function (CDF) [72]. Figure $8(\mathrm{c})$ shows the comparison between the cumulative distribution function of waiting times obtained from numerical simulation of Eqs. (3) and for a Poisson process. Notice that

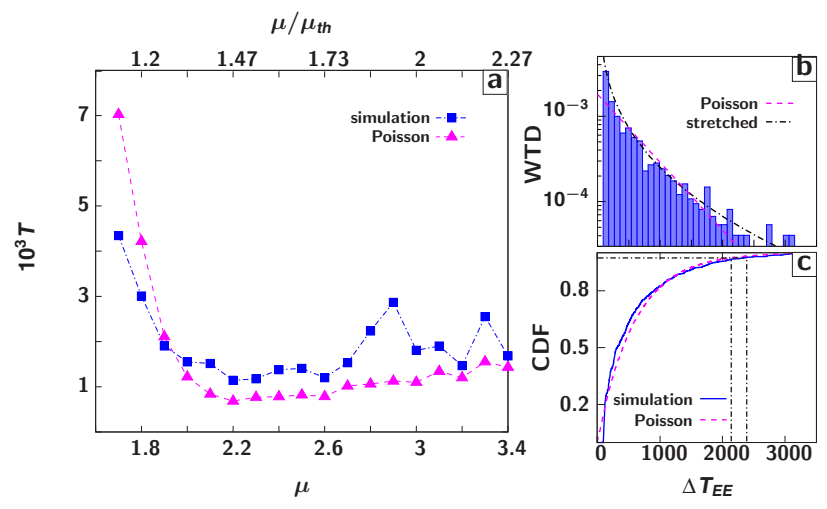

FIG. 8. Statistical characterization of time interval between numerically observed extreme events from Eqs. (3). (a) Maximal time duration $T$ within which a least one extreme event should be observed vs pump parameter. Numerical simulation (squares) compared to an exact Poisson process (triangles) with the same rate of events over $t_{\max }=40000$. (b) Waiting time distribution (WTD) between successive extreme events for $\mu=2.0$. The dashed line is a fit with an exponential distribution and the dash-dotted line is a fit with a stretched exponential with stretching parameter 0.5. (c) Cumulative distribution function (CDF). The horizontal dot-dashed line corresponds to 0.975 and the abscissa of its intersections with the CDFs gives the maximal time duration.

there is a qualitative similarity between these two processes. Moreover, we have compared the maximal inter-arrival time [cf. Fig. 8(a)] versus the pump parameter $\mu$ for both processes. This value is obtained by considering the time $T$ such that the CDF reaches 0.975 [72]. From the above analysis, we conclude that the time difference between extreme events is not a Poisson process, but is well described by a stretched exponential distribution function. The difference with the memoryless Poisson distribution is attributed to correlations in the spatiotemporally chaotic dynamics. This observation is in agreement with the experimental results of the time difference between extreme events for the total intensity analyzed earlier (cf. Fig. 3).

\section{B. Spatiotemporal extended intermittency}

As mentioned earlier, the total intensity of the system exhibits intermittent behavior when the system is in the regime of spatiotemporal chaos [cf. left panel of Fig. 6(d)]. Spatiotemporal chaotic intermittency was intensively studied in the context of the Ginzburg-Landau equation [73,74] and coupled-map lattices [75]. The main feature of this type of spatiotemporal chaotic behavior is to exhibit self-similarity with a Sierpinski carpet structure type. Figure 9 shows the numerical spatiotemporal evolution of the intensity of the intracavity electric-field envelope $|E|$ in the complex spatiotemporal regime. Considering a long record of the temporal evolution of the intracavity field [see Fig. 9(a)], we observe the emergence of a large number of peaks. To emphasize that these peaks correspond to extreme events, small portions of spatiotemporal diagram are extracted in the insets of Fig. 9(a). Clearly, among the complex oscillations in the spatiotemporal diagram, extreme events are observed with a large amplitude 

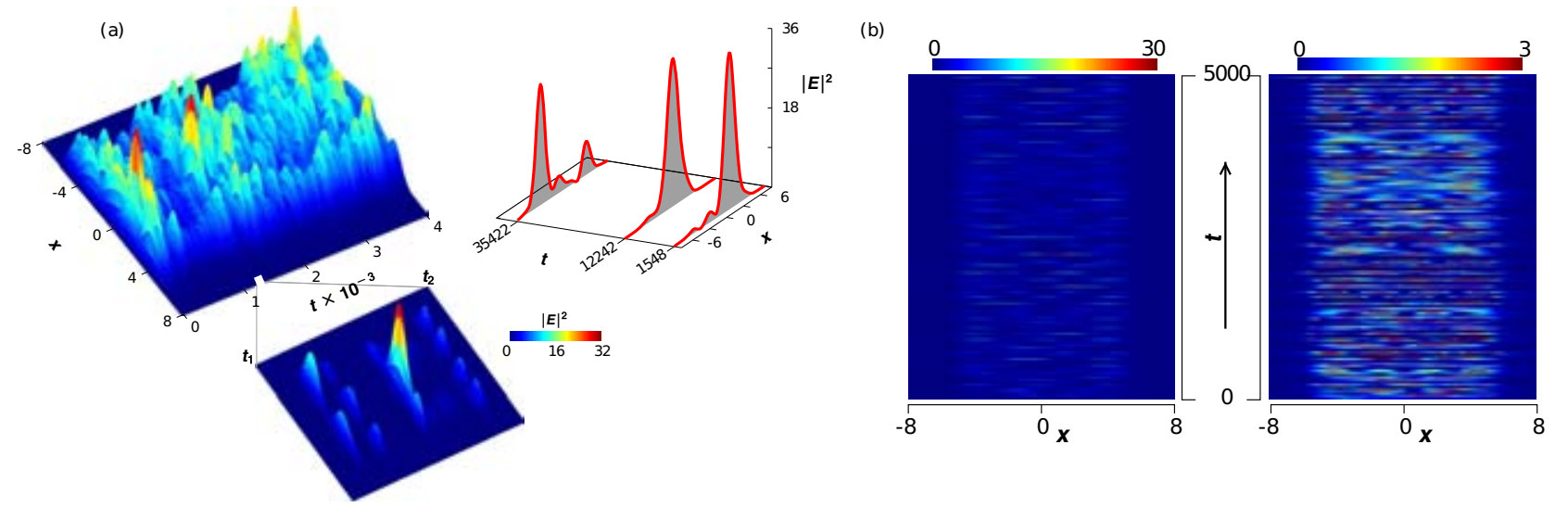

FIG. 9. Spatiotemporal intermittence evolution of the intensity of the intracavity electric-field envelope $E(x, t)$ of a one-dimensional spatially extended laser with a saturable absorber medium, Eq. (3). (a) Long record of the spatiotemporal evolution of the intracavity field. Insets account for excerpts of spatiotemporal diagram and for spatial profile of the three highest intensity peaks observed in the long record. (b) Spatiotemporal evolution plotted in false color with two different color scales in order to highlight the intermittent behavior.

that is several times greater than the average. To understand the complex dynamics exhibited by the spatiotemporal diagrams, we have changed the color scale to monitor the dynamic of modes with large and small amplitudes. Spatiotemporal diagrams with different color scale are illustrated in Fig. 9(b). One can infer that high-amplitude modes are intermittent and, as we have shown previously, they are statistically memoryless. On the other hand, the dynamics of the low-intensity peaks is complex. An obvious self-similar structure is not observed, as is the case of the spatiotemporal intermittency. However, the observed dynamics is intermittent. Hence, we have termed our observed dynamics as spatiotemporal extended intermittency. A deeper characterization of this type of dynamic is in progress.

\section{Statistical spatiotemporal analysis}

From the numerical integration of Eqs. (3) we can also calculate the temporal cross-correlation of the intensity $I(x, t)$ from Eq. (2). The result is shown in Fig. 10. The behavior is very similar to what was observed in the experiment in Fig. 4. There is no sign of propagation of coherent structure is the system, except for very low pump value $\mu=1.6$, very close to laser threshold [Fig. 10(a)]. There is, however, a spatial correlation length clearly visible in the system. The size of the correlation length decreases with pump, as the system dynamics become more complex. A close inspection of the central part of Fig. 10(c) reveals that the temporal cross correlation has positive values before and after the maximum, a feature that may be exploited for predicting extreme events in the short term.

\section{EXTREME EVENTS FOLLOWING BIFURCATION TO SPATIOTEMPORAL CHAOS}

As we have shown in previous sections and in our previous work [41], the appearance of extreme events is strongly related to the emergence of spatiotemporal chaos. To emphasize the above statement, we show in Figs. 11(a) and 11(c) respectively the proportion of extreme events $p_{\mathrm{EE}}$ and the excess kurtosis $\gamma_{2}$ as function of pump power parameter for two different observables: the height of the total intensity across the laser $I_{\text {tot }}$ and the intensity of the spatiotemporal peaks $I_{\text {loc }}$. The excess kurtosis is given by $\gamma_{2}=\mu_{4} / \mu_{2}^{2}-3$ with $\mu_{i}$ the centered moment of order $i$. The proportion of extreme events and the excess kurtosis are strongly correlated in both cases. A natural order parameter describing the change of dynamical behavior is the largest Lyapunov exponent and the KaplanYorke dimension. For stationary solutions, the corresponding largest Lyapunov exponent is negative and the dimension of Kaplan-Yorke is zero. In the case of periodic or quasiperiodic solutions the corresponding largest Lyapunov exponent and the
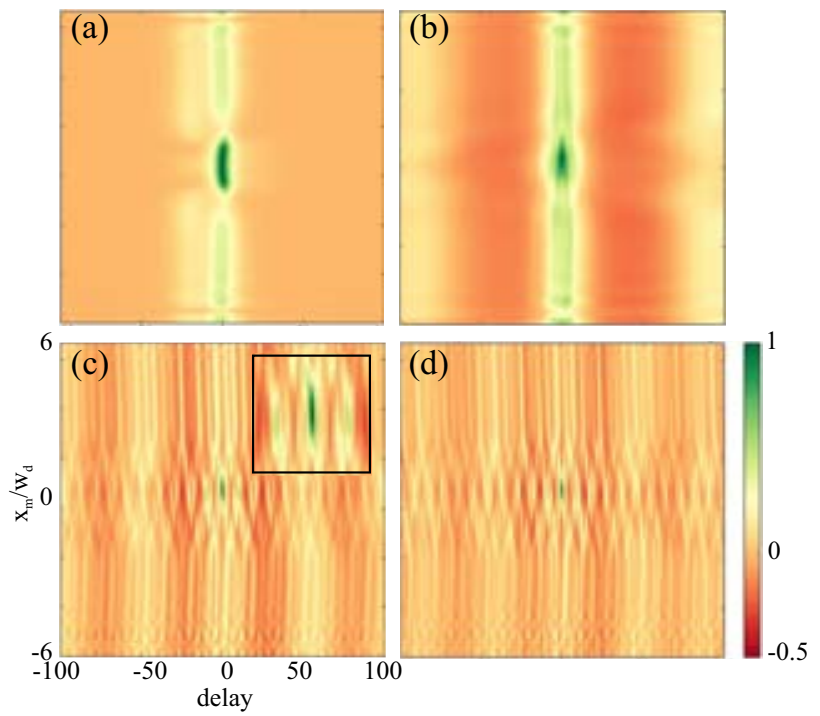

FIG. 10. Numerical cross correlation of the response between the center of the laser (point $\mathrm{C}, x_{m}=0$ ) and a sliding point $\mathrm{M}\left(x_{m}\right)$. Pumping is $\mu=1.6,2.0,2.9,3.4$ [panels (a)-(d), resp.]. In panel (c), an inset shows a closeup of the central part. 

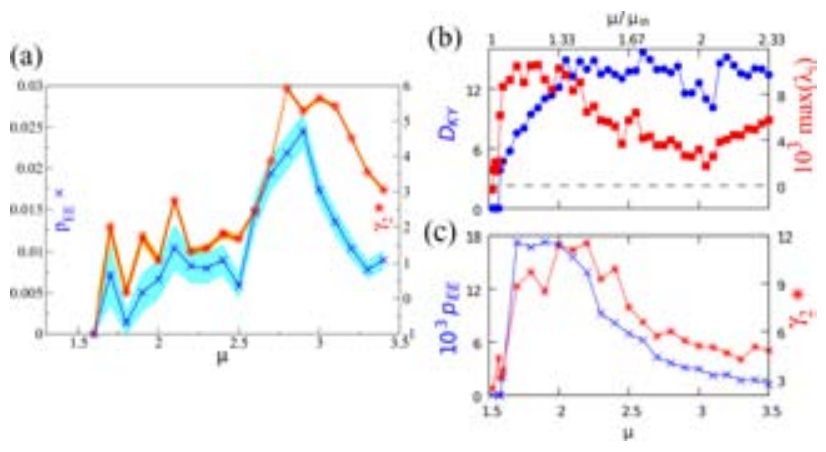

FIG. 11. Emergence of extreme events and spatiotemporal chaos according to different observables. (a) Proportion of extreme events $p_{\mathrm{EE}}(\times)$ and excess kurtosis $\gamma_{2}(*)$ as function of pump parameter $\mu$ considering the height of the total intensity across the laser. (b) Largest Lyapunov exponent $\max \left(\lambda_{i}\right)$ (squares) and Kaplan-Yorke dimension $D_{\mathrm{KY}}$ [circles, from Eq. (1)] as function of pump parameter $\mu$. (c) Proportion of extreme events $p_{\mathrm{EE}}(\times)$ and excess kurtosis $\gamma_{2}(*)$ as function of pump parameter $\mu$ considering the intensity of the local spatiotemporal maxima.

Kaplan-Yorke dimension are both zero. For chaotic dynamical behaviors the corresponding largest Lyapunov exponent and Kaplan-Yorke dimension are strictly positive. Figure 11(b) shows the largest Lyapunov exponent $\max \left(\lambda_{i}\right)$ and KaplanYorke dimension $D_{Y K}$ as function of pump power parameter $\mu$. Looking at Figs. 11(a) and 11(c) we see that whatever the observable considered, extreme events appear only when the Kaplan-Yorke dimension and the maximum Lyapunov exponent are strictly positive. This means that spatiotemporal chaos is a prerequisite to observe extreme events. Moreover, as the spatiotemporal complexity increases sharply with the pump (see the initial increase of $D_{\mathrm{KY}}$ and $\max \left(\lambda_{i}\right)$ with $\mu$ ), the proportion of extreme events and the excess kurtosis grow. There is a maximum in the proportion of extreme events that depends on the observable considered, and then the proportion of extreme events decreases with the pump, in a way which seems not completely correlated neither to the Kaplan-Yorke dimension nor to the maximum Lyapunov dimension. If the initial evolution of $p_{E E}(\mu)$ seems to follow closely the one of the maximum Lyapunov exponent until $\mu \simeq 3.0$, the evolution of both quantities then diverge, as we have checked by comparing both quantities for values of the pump up to $\mu=5$. Therefore, the largest Lyapunov exponent is not totally correlated with the proportion of extreme events. Abrupt variations of the largest Lyapunov exponent or of the Kaplan-Yorke dimension can be a manifestation of instabilities of a chaotic state. A classical example of this behavior for the largest Lyapunov exponent can be found with the logistic model [76]. This phenomenon can generate the spatiotemporal analog of chaos crisis, as theoretically demonstrated in a laser model in Refs. [77,78]. We have looked for this behavior in our data but we could not reliably extract the derivative of the Kaplan-Yorke dimension or of the maximum Lyapunov exponent with respect to the pump. We can, however, conclude from the above results that extreme events in our spatially extended microcavity laser follow bifurcation to spatiotemporal chaos. Now if we compare the two observables $I_{\text {tot }}$ and $I_{\text {loc }}$, we see that in both cases the evolutions of $p_{\mathrm{EE}}$ and of $\gamma_{2}$ are correlated in each case. The maximum proportion of extreme events is also shifted in the same direction with respect to the maximum of the excess kurtosis. An interesting feature lies in the fact that $p_{\text {EE }}$ peaks for a lower value of the pump in the case of the observable $I_{\text {loc }}$. The proportion measured is comparable up to $\mu \sim 2$ and is then very different with opposite trends. We attribute this difference in the fact that for low pump values, the correlation length in the system is large as testified by Fig. 10. Hence, there is hardly more than one large peak in the spatial section that dominates the observable value and gives rise in both cases to extreme events. By contrast, the correlation length decreases if the pump is large; some remaining large peak intensities can add up to form extremes in the total intensity height that are not present in the spatiotemporal maxima. For very large pump both cases agree and a lower proportion of extremes are observed, a consequence of a complex dynamics with few excursions to extreme values.

\section{CONCLUSIONS}

We have investigated experimentally and theoretically the emergence of extreme events in a quasi-1D broad-area laser with a saturable absorber. We have analyzed the physical origin of extreme events that occur because of the onset of deterministic spatiotemporal chaos in the system. Hence, the system exhibits extreme events when the system has qualitative changes that lead to complex dynamical behaviors. We have characterized the chaotic behavior through the calculation of the Lyapunov spectrum, the Kaplan-Yorke dimension, the largest Lyapunov exponent, reconstruction embedding, and frequency analysis. The statistical and dynamic analysis lead us to conjecture that quasiperiodic behavior may exhibit extreme events. Studies in this direction are in progress. In the chaotic regime, we conclude that the time difference between extreme events is close to but not strictly a Poisson process and can be described by a stretched exponential distribution function in the tail of the distribution. Short-term and very long-term correlations in the dynamics explain the deviations from a memoryless Poisson process. From these analyses, we assign to the spatiotemporal chaotic behavior exhibited by the system the mechanism of spatiotemporal extended intermittency.

The theoretical analysis presented in this work is based on deterministic processes. However, it is known that inherent fluctuations in macroscopic multistable systems - noise - can induce extreme events through noise-induced transitions [32]. The parameter region that we have considered in the present study is monostable, which ensures that noise with small intensity level only generates degradation of the observed signal. Numerical simulations of a one-dimensional spatially extended laser with a saturable absorber medium with additive noise, Eqs. (3), present therefore a similar dynamical behavior to that observed in the deterministic case.

Irregular dynamics is obviously a prerequisite for the observation of extreme events, but we show that the proportion of extreme events is directly linked to the evolution of the Kaplan-Yorke dimension and largest Lyapunov exponent. A higher dimensional dynamics does not necessarily lead to a higher number of extreme events. The origin of extreme 
events in that case is thus to be found in the nature of the spatiotemporal complexity that takes place, and thus it could offer interesting prospects for applications to control them by changing the system geometry or the nature of the coupling.

\section{ACKNOWLEDGMENTS}

M.G.C. is thankful for the financial support of FONDECYT Project No. 1150507. The authors acknowledge partial support from the French network Renatech and the ANR Blanc project Optiroc.
[1] G. Nicolis and I. Prigogine, Self-Organization in Nonequilibrium Systems, A Wiley-Interscience Publication (Wiley, New York, 1977).

[2] L. M. Pismen, Patterns and Interfaces in Dissipative Dynamics (Springer, Berlin, 2006).

[3] M. Cross and H. Greenside, Pattern Formation and Dynamics in Nonequilibrium Systems (Cambridge University Press, New York, 2009).

[4] M. I. Rabinovich, A. Ezersky, and P. D. Weidman, The Dynamics of Patterns (World Scientific, Singapore, 2000).

[5] P. Coullet and J. Lega, Europhys. Lett. 7, 511 (1988).

[6] P. Coullet, L. Gil, and J. Lega, Phys. Rev. Lett. 62, 1619 (1989).

[7] G. Goren, J.-P. Eckmann, and I. Procaccia, Phys. Rev. E 57, 4106 (1998).

[8] Y. Kuramoto, Chemical Oscillations, Waves, and Turbulence, Springer Series in Synergetics, Vol. 19 (Springer-Verlag, Berlin Heidelberg, 2012)

[9] W. Decker, W. Pesch, and A. Weber, Phys. Rev. Lett. 73, 648 (1994).

[10] B. Echebarria and H. Riecke, Phys. Rev. Lett. 84, 4838 (2000).

[11] M. A. Miranda and J. Burguete, Phys. Rev. E 79, 046201 (2009).

[12] P. Brunet and L. Limat, Phys. Rev. E 70, 046207 (2004).

[13] Q. Ouyang et al., Nature (London) 379, 143 (1996).

[14] Z. Qu, J. N. Weiss, and A. Garfinkel, Phys. Rev. Lett. 78, 1387 (1997).

[15] S.-Q. Zhou and G. Ahlers, Phys. Rev. E 74, 046212 (2006).

[16] S. J. Moon, M. D. Shattuck, C. Bizon, D. I. Goldman, J. B. Swift, and H. L. Swinney, Phys. Rev. E 65, 011301 (2001).

[17] N. Verschueren, U. Bortolozzo, M. G. Clerc, and S. Residori, Phys. Rev. Lett. 110, 104101 (2013).

[18] N. Verschueren, U. Bortolozzo, M. Clerc, and S. Residori, Philos. Trans. R. Soc. London, Ser. A 372, 20140011 (2014).

[19] M. G. Clerc, G. González-Cortés, V. Odent, and M. Wilson, Opt. Express 24, 15478 (2016).

[20] G. Nicolis and C. Nicolis, Foundations of Complex Systems: Emergence, Information, and Predicition, 2nd ed. (World Scientific, Singapore, 2012).

[21] M. Onorato, S. Residori, U. Bortolozzo, A. Montina, and F. Arecchi, Phys. Rep. 528, 47 (2013).

[22] C. Kharif and E. Pelinovsky, Eur. J. Mech. B 22, 603 (2003).

[23] D. R. Solli, C. Ropers, P. Koonath, and B. Jalali, Nature (London) 450, 1054 (2007).

[24] J. M. Dudley, G. Genty, and B. J. Eggleton, Opt. Express 16, 3644 (2008).

[25] A. Mussot, A. Kudlinski, M. Kolobov, E. Louvergneaux, M. Douay, and M. Taki, Opt. Express 17, 17010 (2009).

[26] B. Kibler, J. Fatome, C. Finot, G. Millot, F. Dias, G. Genty, N. Akhmediev, and J. M. Dudley, Nat. Phys. 6, 790 (2010).

[27] F. T. Arecchi, U. Bortolozzo, A. Montina, and S. Residori, Phys. Rev. Lett. 106, 153901 (2011).
[28] A. Antikainen, M. Erkintalo, J. M. Dudley, and G. Genty, Nonlinearity 25, R73 (2012).

[29] S. Birkholz, E. T. J. Nibbering, C. Brée, S. Skupin, A. Demircan, G. Genty, and G. Steinmeyer, Phys. Rev. Lett. 111, 243903 (2013).

[30] D. Pierangeli, F. Di Mei, C. Conti, A. J. Agranat, and E. DelRe, Phys. Rev. Lett. 115, 093901 (2015).

[31] C. Lecaplain, P. Grelu, J. M. Soto-Crespo, and N. Akhmediev, Phys. Rev. Lett. 108, 233901 (2012).

[32] A. N. Pisarchik, R. Jaimes-Reátegui, R. Sevilla-Escoboza, G. Huerta-Cuellar, and M. Taki, Phys. Rev. Lett. 107, 274101 (2011).

[33] C. Bonatto, M. Feyereisen, S. Barland, M. Giudici, C. Masoller, J. R. Leite, and J. R. Tredicce, Phys. Rev. Lett. 107, 053901 (2011).

[34] A. Montina, U. Bortolozzo, S. Residori, and F. T. Arecchi, Phys. Rev. Lett. 103, 173901 (2009).

[35] S. Randoux and P. Suret, Opt. Lett. 37, 500 (2012).

[36] M. G. Kovalsky, A. A. Hnilo, and J. R. Tredicce, Opt. Lett. 36, 4449 (2011).

[37] M. G. Clerc, G. González-Cortés, and M. Wilson, Opt. Lett. 41, 2711 (2016).

[38] A. K. D. Bosco, D. Wolfersberger, and M. Sciamanna, Opt. Lett. 38, 703 (2013).

[39] V. Odent, M. Taki, and E. Louvergneaux, Nat. Hazards Earth Syst. Sci. 10, 2727 (2010).

[40] N. Marsal, V. Caullet, D. Wolfersberger, and M. Sciamanna, Opt. Lett. 39, 3690 (2014).

[41] F. Selmi, S. Coulibaly, Z. Loghmari, I. Sagnes, G. Beaudoin, M. G. Clerc, and S. Barbay, Phys. Rev. Lett. 116, 013901 (2016).

[42] S. Barbay, Y. Ménesguen, I. Sagnes, and R. Kuszelewicz, Appl. Phys. Lett. 86, 151119 (2005).

[43] T. Elsass, K. Gauthron, G. Beaudoin, I. Sagnes, R. Kuszelewicz, and S. Barbay, Eur. Phys. J. D 59, 91 (2010).

[44] M. G. Clerc and N. Verschueren, Phys. Rev. E 88, 052916 (2013).

[45] P. Manneville, in Chaos? The Interplay between Stochastic and Deterministic Behaviour (Springer, New York, 1995), pp. 257272.

[46] E. Ott, Chaos in Dynamical Systems (Cambridge University Press, Cambridge, UK, 2002).

[47] H. Abarbanel, Analysis of Observed Chaotic Data (Springer, New York, 2012).

[48] D. Ruelle, Commun. Math. Phys. 87, 287 (1982).

[49] M. R. Paul, M. I. Einarsson, P. F. Fischer, and M. C. Cross, Phys. Rev. E 75, 045203 (2007).

[50] T. Elsass, K. Gauthron, G. Beaudoin, I. Sagnes, R. Kuszelewicz, and S. Barbay, Appl. Phys. B 98, 327 (2010).

[51] S. Barbay, R. Kuszelewicz, and J. R. Tredicce, Adv. Opt. Tech. 2011, 628761 (2011). 
[52] R. Kuszelewicz, S. Barbay, G. Tissoni, and G. Almuneau, Eur. Phys. J. D 59, 1 (2010).

[53] S. Barbay, R. Kuszelewicz, and A. M. Yacomotti, Opt. Lett. 36, 4476 (2011).

[54] F. Selmi, R. Braive, G. Beaudoin, I. Sagnes, R. Kuszelewicz, and S. Barbay, Phys. Rev. Lett. 112, 183902 (2014).

[55] J. P. Eckmann and D. Ruelle, Rev. Mod. Phys. 57, 617 (1985).

[56] Y. Pomeau and P. Manneville, Commun. Math. Phys. 74, 189 (1980).

[57] S. Birkholz, C. Brée, I. Veselić, A. Demircan, and G. Steinmeyer, Sci. Rep. 6, 35207 (2016).

[58] V. Lucarini, D. Faranda, A. de Freitas, J. de Freitas, M. Holland, T. Kuna, M. Nicol, M. Todd, and S. Vaienti, Extremes and Recurrence in Dynamical Systems, Pure and Applied Mathematics: A Wiley Series of Texts, Monographs, and Tracts (Wiley, New York, 2016).

[59] A. Bunde, J. F. Eichner, S. Havlin, and J. W. Kantelhardt, Phys. A (Amsterdam, Neth.) 330, 1 (2003).

[60] E. G. Altmann and H. Kantz, Phys. Rev. E 71, 056106 (2005).

[61] P. Sibani and P. B. Littlewood, Phys. Rev. Lett. 71, 1482 (1993).

[62] M. Bache, F. Prati, G. Tissoni, R. Kheradmand, L. Lugiato, I. Protsenko, and M. Brambilla, Appl. Phys. B 81, 913 (2005).

[63] W. Chow, S. Koch, and M. Sargent, Semiconductor-Laser Physics (Springer-Verlag, Berlin, 1994).

[64] E. Hairer and G. Wanner, Solving Ordinary Differential Equations II: Stiff and Differential-Algebraic Problems (Springer, Berlin, 1996).
[65] J. L. A. Dubbeldam and B. Krauskopf, Opt. Commun. 159, 325 (1999).

[66] A. E. Siegman, Lasers (University Science Book, Sausalito, CA, 1986).

[67] E. A. Jackson, Perspectives of Nonlinear Dynamics, Vol. 2 (Cambridge University Press, Cambridge, 1990).

[68] J. M. Dudley, F. Dias, M. Erkintalo, and G. Genty, Nat. Photon 8, 755 (2014).

[69] C. Rimoldi, S. Barland, F. Prati, and G. Tissoni, arXiv: 1610.06452.

[70] F. Christiansen and H. H. Rugh, Nonlinearity 10, 1063 (1997).

[71] T. J. Bridges and S. Reich, Phys. D (Amsterdam, Neth.) 156, 219 (2001)

[72] J. E. Gentle, Computational Statistics, Vol. 308 (Springer, New York, 2009).

[73] H. Chaté and P. Manneville, Phys. Rev. Lett. 58, 112 (1987).

[74] H. Chate, Nonlinearity 7, 185 (1994).

[75] H. Chaté and P. Manneville, Phys. D (Amsterdam, Neth.) 32, 409 (1988).

[76] E. A. Jackson, Perspectives of Nonlinear Dynamics, Vol. 1 (Cambridge University Press, Cambridge, 1989).

[77] C. Metayer, A. Serres, E. J. Rosero, W. A. S. Barbosa, F. M. de Aguiar, J. R. R. Leite, and J. R. Tredicce, Opt. Express 22, 19850 (2014).

[78] N. M. Granese, A. Lacapmesure, M. B. Agüero, M. G. Kovalsky, A. A. Hnilo, and J. R. Tredicce, Opt. Lett. 41, 3010 (2016). 